\title{
Application of one kind of training equipment in maintenance teaching
}

\author{
Kan Liu' ${ }^{1,}$, Xinxin Tian ${ }^{2, b}$, Yuan Lin ${ }^{1, c}$ \\ ${ }^{1}$ Department of Guidance, Wuhan Mechanical Technology College, Wuhan, 430075, China \\ ${ }^{2}$ Department of Computer, Wuhan Mechanical Technology College, Wuhan, 430075, China \\ aemail: 13886058175@163.com, bemail:Ik820727@sina.com, cemail:31301551@qq.com
}

Keywords: Training device; Maintenance teaching; Vocational Education

\begin{abstract}
In order to carry out Vocational Education, strengthen the students' post office ability, improve the teaching effect, we develop a maintenance training device. The device used in the form of semi physical simulation, can set up the simulation of the fault, for maintenance training, is a set of teaching, training, maintenance in one of the simulation teaching platform. The practice has proved that the application of this device can stimulate the students' learning enthusiasm, improve the students' participation, and cultivate the students' ability of equipment maintenance, and achieve the expected teaching effect.
\end{abstract}

\section{Introduction}

With the development of Vocational Education, the students' practical ability of higher requirements, the requirements of teaching is also gradually increased. ${ }^{[1]}$

The Launch Control Equipment(LCE) is the core of XX Equipment. LCE is composed of electronic components, compact structure and high integration. The following problems exist in the maintenance teaching:

a) insufficient training conditions

The detection and maintenance of LCE is more complex, and the lack of teaching conditions, it is difficult to effectively train students' equipment maintenance ability.

b) training low efficiency cost ratio

In the past, we need to make the signal cable, connect the signal source, welding circuit board, demonstration equipment maintenance. The equipment is easy to damage, resulting in high training cost and low efficiency.

c) fault simulation is not true

Real fault is difficult to simulate, the existence of fault is not realistic.

In order to solve the above problems, improve the students' ability of equipment inspection and maintenance, we have developed this kind of maintenance training device(Device FK).

\section{The function, structure and characteristics of Device FK}

a) the main function of Device FK

Device FK is mainly used for the maintenance of training, training students in the maintenance of thinking and maintenance skills, improve the students' ability of circuit analysis and maintenance. Students can better complete the work of equipment maintenance.

b) Structure of equipment

The structure of the device uses a microcontroller, CPLD, WIFI, configuration software, etc.. When the equipment works, the software controls the hardware to simulate the setting up of the fault, and constructs the teaching and training platform of the integrated teaching, training and maintenance. ${ }^{[2]}$

The equipment is made up of Main device and Slave device. Main device is made up of computer and wireless router. Slave device is made up of touch computer, PLC controller, special signal source, circuit board of fault simulation, signal testing unit, combination of connector and a power module. The relationship of each part of the Device FK is shown in Figure 1. 

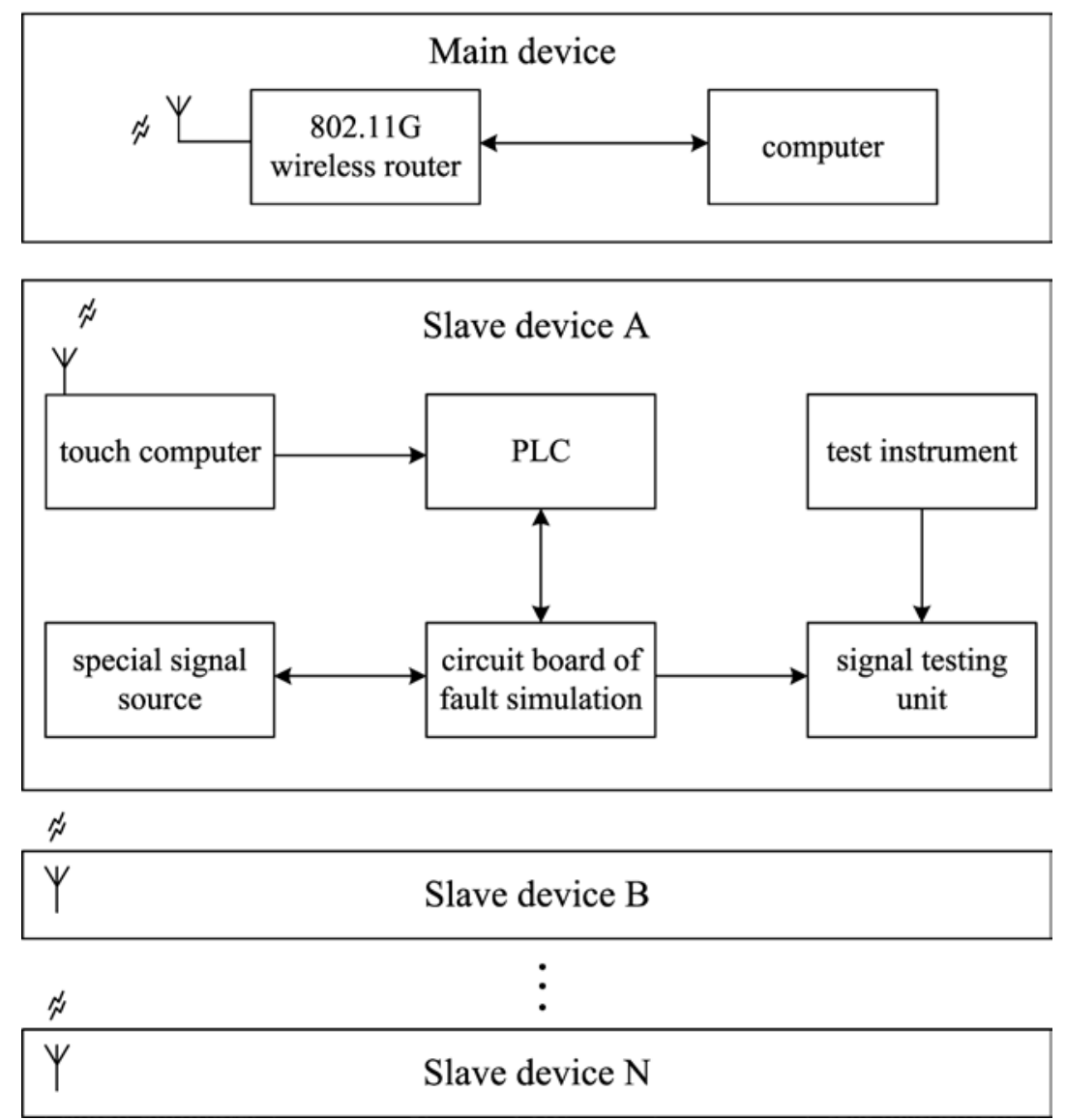

Figure 1 Device architecture diagram

The teacher sets the fault on the Main device, and sends the information to the Slave device. The slave device and the PLC interface are provided with a failure. The students can see the circuit of the transmitting device in the software interface, observe the failure phenomenon and analyze the causes of the failure. The software interface is shown in Figure 2. Students use the test instrument to measure the electrical signal of each point, and try to eliminate the trouble on the software interface of the slave device. At the same time, the Slave device troubleshooting through WIFI network back to the Main device. Main device record data, evaluation results. 


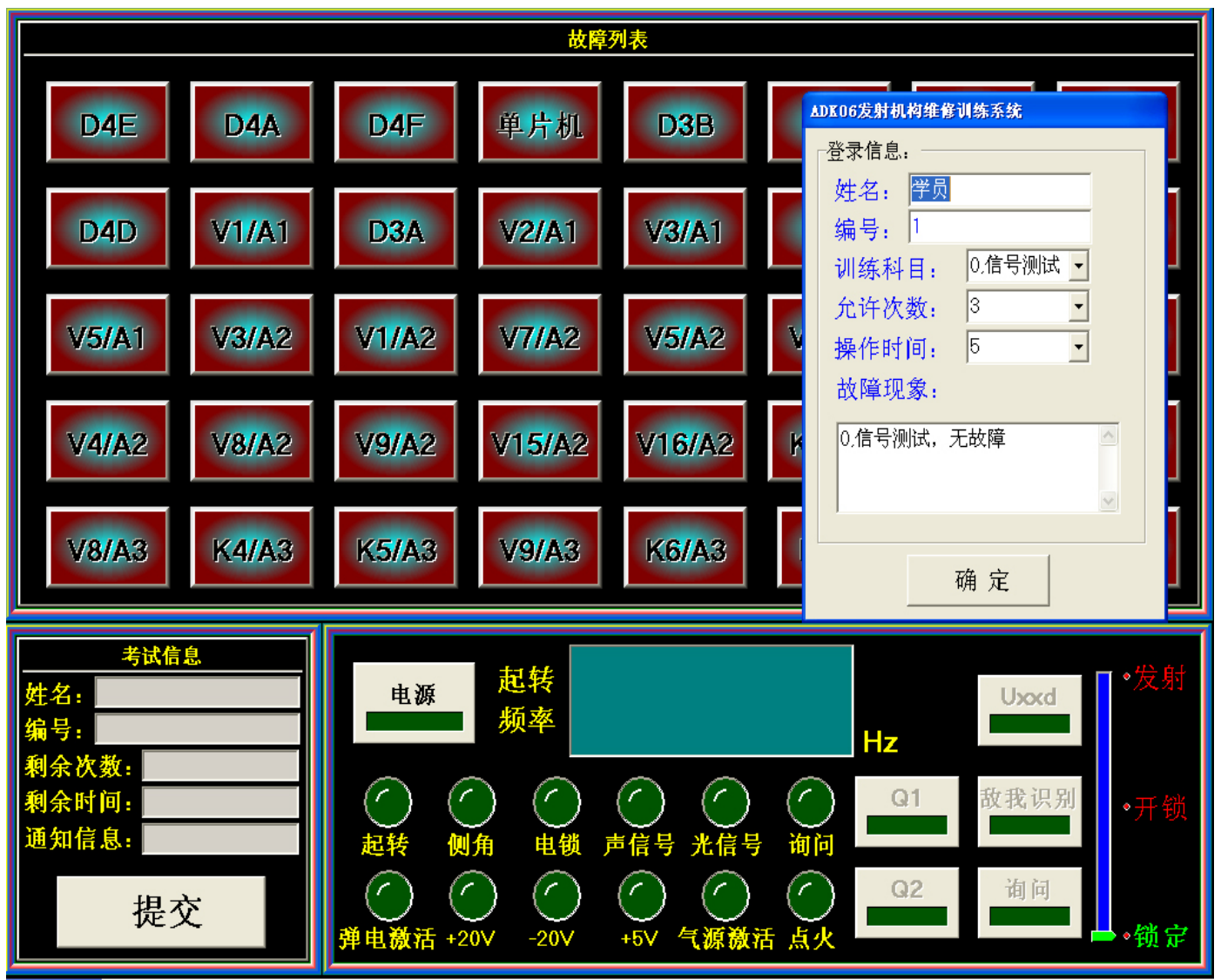

c) Characteristics of equipment ${ }^{[3 \sim 4]}$

Figure 2 Software interface

Device FK used in the simulation of maintenance, for the LCE maintenance training provides a new method.

Device FK uses the form of semi physical simulation, signal testing unit layout components, signal relation and SMT completely consistent, ensure maintenance training environment more realistic.

Students through the use of Device FK, can better understand the work principle of LCE, faster control of detect and repair method, effectively improve the students' ability to repair LCE.

Device FK can maximally reduce equipment loss and reduce the fault time and set up fault exclusion time, effectively improve the efficiency of maintenance training, greatly reduce the cost of maintenance training.

\section{Effect of teaching application}

The purpose of Vocational Education is to cultivate students' practical ability. The equipment is close to the teaching of equipment, which is suitable for the teaching of principle, the demonstration of the fault, the fault analysis and the trouble shooting training, which meets the needs of theory teaching and practical teaching.

a) To meet the teaching, theory and practice linked

Device FK can truly show the working environment of the LCE, provide real-time test interface, and can easily test the signal and display all kinds of signal waveforms. The theory and practice of the link, to stimulate the students' learning interest, enhance the students' perceptual knowledge, deepen the understanding of the circuit principle. Use process as shown in Figure 3. 


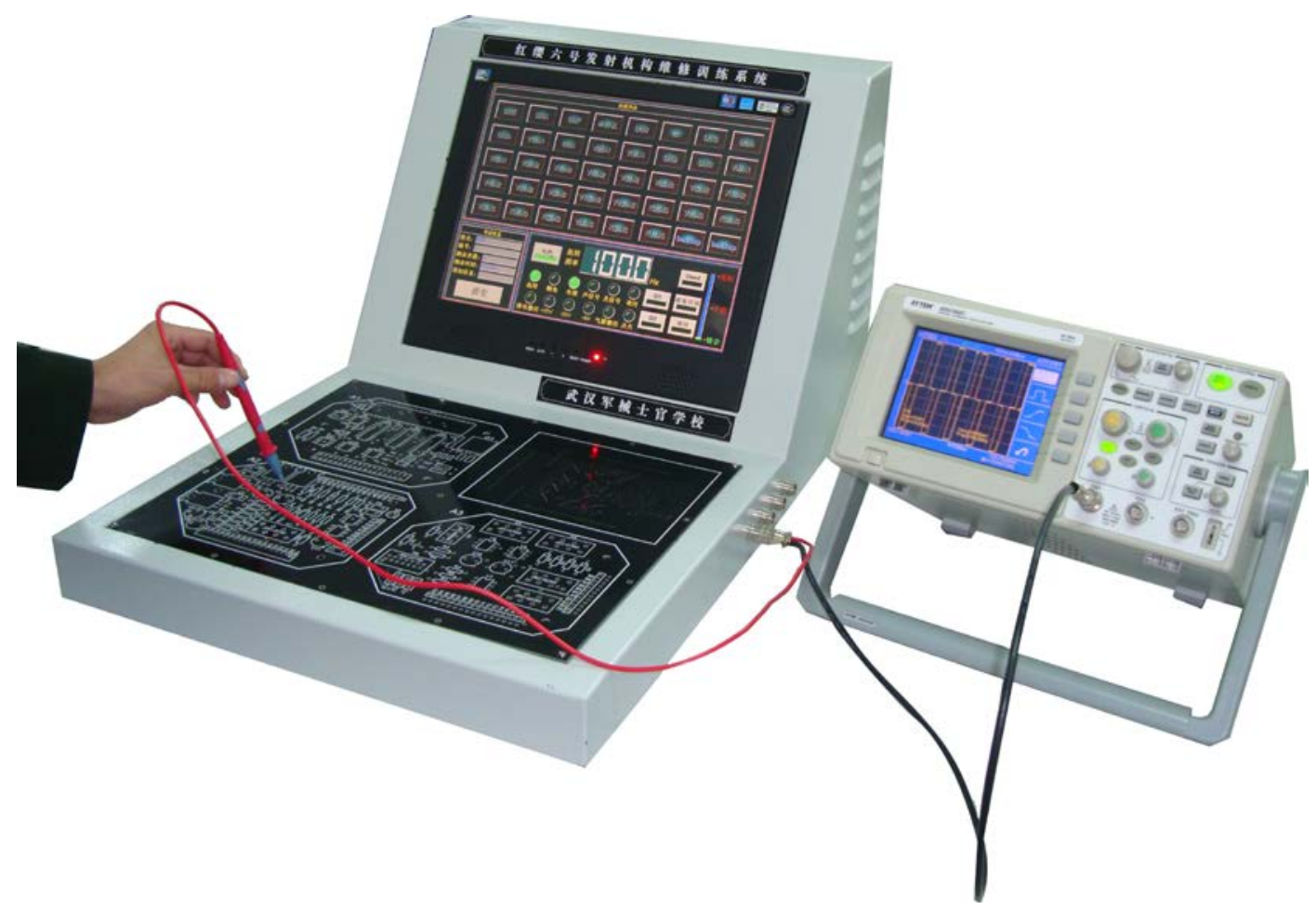

Figure 3 LCE use

b) Abundant maintenance training means, increase the number of maintenance training group

At present, the development of a variety of models Device FK, to meet the needs of various types of LCE maintenance training. Device FK is a high degree of integration, small occupied space. We overcome the difficulty of the equipment, increase the number of maintenance training group, enrich the maintenance training means, reduce the ground and equipment needs.

c) Provide a variety of fault types, improve the efficiency of unit time training

Device FK can simulate all the faults of LCE, and can also set up many kinds of faults, and enrich the fault types. In the training, eliminating the need for LCE disassembly and fault setting time, improve the efficiency of unit time training.

\section{Conclusion}

Device FK by real LCE real fault, provide a realistic maintenance training environment, improve the students' training participation, stimulate the students' learning interest, teaching effect has been greatly improved. This system is not only suitable for the theory and practice teaching of the relevant majors in Colleges and universities, but also can be used in the maintenance personnel training.

\section{Acknowledgement}

In this paper, the research was sponsored by the Teaching Reform Research Project of Wuhan Mechanical Technology College (Project No. 2015-55).

\section{References}

[1] Zemin Deng, Guochun Han. Vocational Education training design [M]. China Railway Press, 2008.

[2] Wen Lu. Non immersive virtual maintenance simulation platform development [D]. Wuhan University of Technology, 2010.

[3] Yong Shan. Research and implementation of key technologies of real time semi physical simulation platform [D]. National University of Defense Technology, 2010. 
[4] Yan Zhou, Jianwei Dai. HLA Simulation Program Design [M]. Electronic Industry Press, 2002. 